\title{
Study on Road Condition Recognition Method Based on Convolutional Neural Network
}

\author{
Feng Jiang ${ }^{1, \text { a) }}$, Ning $\mathrm{Wei}^{1}$, Yan Zhou ${ }^{1}$ and Hengjun $\mathrm{Niu}^{2}$ \\ ${ }^{1}$ Academy of army armored force, Beijing 100072, China. \\ ${ }^{2}$ Jinzhu Materials Technology Co. Ltd, Shandong 251109, China. \\ a) Corresponding author: onlywer@foxmail.com
}

\begin{abstract}
In recent years, frequent traffic accidents, traffic accidents captured countless people's lives, so the road safety problem attracts more and more attention of common people. This paper studies the problem of automatic recognition of road condition, the purpose is to improve the traffic information detection accuracy and robustness. In general, the automatic identification of vehicle targets is vulnerable to all kinds of weather, such as rainy weather, snow weather and sandstorm weather which will weaken the radar target visibility to the public, so it has potential danger. For a long time, the situation caused by this natural deterioration of road safety has not been effectively resolved, or that there is no substantive solution, this is obviously an embarrassing question. From a certain extent, can accurately and effectively carry out the automatic identification of traffic information, thereby reducing the incidence of traffic accidents, but also a symbol of a country's comprehensive strength. In order to avoid the traditional target recognition characteristics easily affected by bad weather caused instability and robustness is not good, in order to overcome this difficulty, we propose a method of road object recognition based on convolution neural network. A large number of experimental results show that this method is very effective, has a high recognition rate, but also has good robustness; the simulation result is a good proof. This method has good adaptability to harsh natural weather, can overcome these unfavorable factors, believe that this method for the implementation of the project to a great extent guide convenience, this is a very meaningful recognition method for the road of the goal.
\end{abstract}

Key words: Road Condition Recognition; Deep Learning; Convolutional Neural Network.

\section{INTRODUCTION}

In recent years, with good reflect the superiority of the socialist system; people's living standards have been significantly improved. Many families have solved eat embarrassment, strong evidence for the improvement of the standard of living is more and more families have the ability to buy some modern equipment. Such as cars [1], trams, motorcycle and car etc... But there will be a serious social problem that is the road safety problem; this massive traffic safety has become a focus of public concern. The reason is that the driver is not enough to concentrate or say the driver for the road of unskilled, or the driver cannot be of man-made and natural disasters are reasonable and accurate and effective to control [2].

The reason why the above situation this is also from the side that we for traffic safety accident control and supervision is not in place, we also illustrate the urgent need to give a complete solution to solve the problem of traffic safety [3]. On the one hand, more and more people have been making unremitting efforts; put forward many constructive opinions and suggestions. For example, Liu Zhan and Wang Zhuhai put forward the vehicle target recognition method of machine learning based on feedback, using the wavelet method to improve the ability of vehicle information recognition, improve its accuracy and robustness [4]; Gong Pinged et al. Proposed a multi sensor information fusion wheel mobile robot target recognition based on mixed traffic Automatic recognition method, a new method of using support vector machine to road traffic, also have good performance [5]. There are a lot of people have made valuable comments, here no longer detailed described one [6]. But this method has 
improved the road condition identification has some defects more or less. The reason is because of its robustness of these algorithms is put aside, but in the aspect of efficient is just passable, is far from satisfactory. It can be said that these algorithms is not intelligent, fast enough [7].

In this paper, we propose a method based on convolutional neural network, which can be applied in automatic recognition of road traffic. This method is very fast, perfect to solve the problem of efficiency.

\section{CONVOLUTIONAL NEURAL NETWORK}

Convolutional neural network is a kind of intelligent algorithm; it is generally used in pattern recognition. This algorithm is well known, with high efficiency, simplicity, scalability, easy operation and good applicability inseparable, below we have a detailed history with this intelligent algorithm and its advantages. Back to its origin, up to 1962, the biologist Hubel and Wiesel two through a new chapter of animal visual cortex opens the research of pattern recognition [8]. They found the animal visual cortex in the existence of a more complex structure than other cell cells, these cells are sensitive to the spatial layout of the outside world, it can in a way we never saw a process of outside space layout, thus the use of visual cortex function on the outside of a target the degree of recognition, it is from the perspective of the pattern recognition began its reign. According to this characteristic, people have done a lot of experiments, training numerous samples to explore the mysteries of the period of pattern recognition. After several generations of unremitting efforts, after the update of a generation algorithm, people finally obtained more ability to adapt to new problems of the algorithm; this is the convolution neural network, referred to as CNN [9].

The essence of convolutional neural network is a multilayer volume contains a number of convolution kernel. Compared with the traditional pattern recognition algorithm for convolutional neural network this algorithm has many advantages [10]. First of all, compared with the traditional pattern recognition algorithm, the parameters of convolutional neural network can be used for training less, the attendant is to reduce the degree of over fitting, reduce the complexity of the network model, so the problem of a large part of the convolutional neural network has good applicability; secondly, compared with the traditional pattern recognition Don't count the convolution algorithm, the neural network neurons use are very few [11]. It makes the space more translation invariance, the resulting benefit is that this method has good robustness for the majority of the pending problems; and convolutional neural network pattern recognition with respect to the traditional algorithm, it is more scalable, can be used in deeper layers [12]; finally, compared to the traditional the pattern recognition of convolutional neural network operation and easy to operate more. The following diagram is presented convolution layer and pool layer convolution neural network [13].
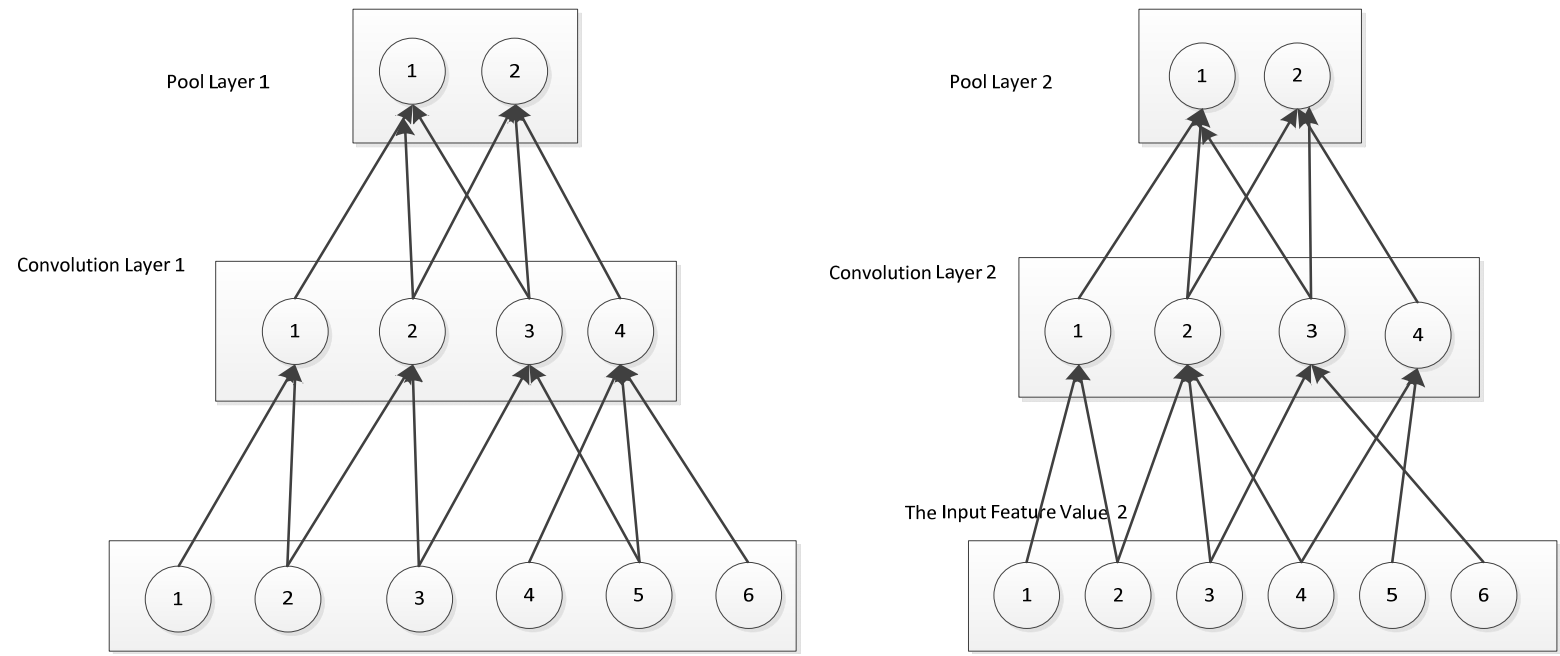

FIGURE 1. The convolution layer and pool layer. 


\section{CONVOLUTIONAL NEURAL NETWORK IS APPLIED TO ROAD CONDITION RECOGNITION}

The model of the convolutional neural network applied to the ROMA database [14]. ROMA is a database of numerical images easily usable to evaluate in a systematic way the performance of road markings extraction algorithms. It comprises more than 100 original images of diverse road scenes, taken in a view point close to the one of the vehicle's driver. Each original image comes with a reference image, build manually, which indicates the positions of the visible road markings.

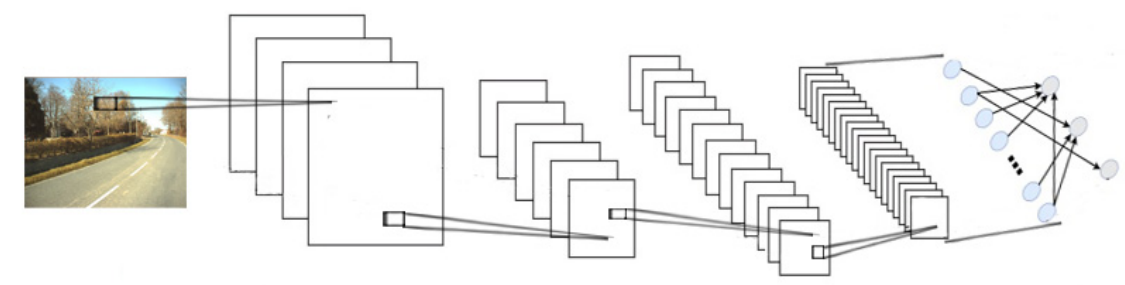

FIGURE 2. The principle of automatic recognition.

The experiment uses the convolution neural network structure as shown in figure 8. Use the Mahout activation function, the network model of growth for the nine layer, the six-layer feature extraction stage, followed by volume (C1) - layer sampling layer (S1) - Mahout layer (M1) - (C2) - convolution layer sampling layer (S2) - Mahout (M2), corresponding to each feature map layer according to the number of times for the $12-6-6-24-12-12$. Feature extraction layer after the data into the classifier, classifier by two fully connected layers, then the classification results are output from the output layer. Table 1 is the model structure of convolutional neural network parameters of the experimental design.

TABLE 1. Convolutional neural network structure parameter table.

\begin{tabular}{|c|c|c|}
\hline layer & type & size/ parameter \\
\hline input & grayscale & $28 * 28$ \\
\hline $1 \mathrm{st}$ & convolution & $5 * 5$ \\
\hline 2nd & mahout & 2 \\
\hline $3 \mathrm{rd}$ & stochastic pooling / max pooling & $2 * 2$ \\
\hline 4th & convolution & $5 * 5$ \\
\hline 5 th & mahout & 2 \\
\hline 6 th & stochastic pooling / max pooling & $2 * 2$ \\
\hline 7 th & dropout & random \\
\hline output & --- & --- \\
\hline
\end{tabular}

This convolution neural network model was trained on ROMA database, the network training period, fast convergence, but fluctuate slightly. In the network training stage, the network has gradually stabilized, there is no significant fluctuations in the error rate detection and recognition, can think of the network to achieve the best training effect at present, namely the network convergence. Detection error using convolutional neural network model improved the rate is lower than the traditional recognition methods. 


\section{CONCLUSION}

In this paper, road condition recognition based on convolutional neural networks, extraction using mahout neurons respectively in the feature, and joined the dropout improvement in the classification stage, experiments on the ROMA database. The experimental results show that the pavement condition identification using convolutional neural network model improved the rate is high, the method can improve the generalization ability of the network, effectively prevent over fitting.

\section{REFERENCES}

1. H. Co-operative transport management with EUROP-SCOUT. Advanced Technology for Road Transport: IVHS and ATT [M]. Ian Catlin, ed. Attach House, MA2004:235-238.

2. Hag R. Locomotive Final Report, Mobil it tunes Transport Project (MTV- locomotive) [M]. BMW AG Munich, Germany, Jerker2000:145-151.

3. Pour Kumises Shah. Evaluation Mobility Impacts of Advanced Information Systems [J]. Journal of Transportation Engineering, May/June, 2004:212-220.

4. H. Traffic Estimation from Location Tracking Data in the Mobile Cellular Network [C]. In: Proc. Wireless Communications and Networking Conference, 2000, 3:1107-1112.

5. Chris Dane, Jean-Luc Grace. Cellular telecommunication and transportation convergence [C] Proceedings, 2001In: Proc. 2001 IEEE Intelligent Transportation System Conference8' 25-29.

6. J M Collator, C - A de la Mescalero, J M Arming. Model based vehicle detection for intelligent Vehicles [C].2004 IEEE Intelligent Vehicles Symposium University of Parma, June 14-17, 2004:572-577.

7. YU X Y, CHEN T J, BAI Y B, et al. Situation assessment method based on rough-vague sets and D-S evidence theory $[\mathrm{C}] / / 4$ th International Conference on Software Engineering and Service Science (ICSESS). USA: IEEE, 2013:746-749.

8. CHE Hokum, LU Fuzztail, Xiang Zhan in. Defects identification by SVM-DS fusion decision-making with multiple features [J]. Chinese Journal of Mechanical Engineering, 2010, 46 (16):101-105.

9. ROSLI M F, HEE I. M, SALMAN I. M, integration of artificial intelligence into dumpsites sharer theory; A review on decision making in condition monitoring[J]. Applied Mechanics Materials, 2015, 773/774:154-157.

10. PLATT. Probabilistic outputs for support vector machines and comparisons to regularized likelihood methods [J]. Advances in Large Margin Classifiers, 2000, 10: 61.

11. H. Co-operative transport management with EUROP-SCOUT. Advanced Technology for Road Transport: IVHS and ATT [M]. Ian Catlin, ed. Attach House, MA2004:235-238.

12. Han J, Cambers M, Pei J. Data mining: concepts and techniques [M].2006. 227-336.

13. Wang W, Yang J, Kuntz R. STING: A statistical information grid approach to spatial data mining[C]. VLDB. 1997. 186-195.

14. T. Viet, J.-P. Tare, P. Nicolle and P. Char bonnier, "Evaluation of Road Marking Feature Extraction", in Proceedings of 11 th IEEE Conference on Intelligent Transportation Systems (ITSC'08), Beijing, China, October 12-15, 2008. 